\title{
Inaugural editorial for integrative obesity and diabetes
}

\author{
Masayoshi Yamaguchi* \\ Department of Hematology and Medical Oncology, Emory University School of Medicine, Atlanta, USA
}

I am very pleased to prepare inaugural editorial for a new Journal "Integrative Obesity and Diabetes (IOD)". Integrative Obesity and Diabetes is an Open Access publication journal, peer reviewed, scholarly journal dedicated to publish articles in all areas of endocrinology, obesity and diabetes. Journal gives information and aims to publish quality papers on endocrinology, obesity and diabetes and their related diseases. The main scope of Journal includes the complete study of pathogenesis, molecular and cellular aspects, metabolic pathway, diagnosis, treatment, clinical management, epidemiology, complications prevention and novel techniques. Journal aims to provide a forum for researchers, physicians and other healthcare professionals to find most recent advances in the areas of obesity and diabetes.

Obesity and diabetes are the most common metabolic disorder associated with many complications. The condition develops due to abnormalities in eating, carbohydrate metabolism and insulin synthesis. The journal is also focused on cutting edge research in the prevention, pharmacological treatment, management, and education of diabetes and related disorders. Integrative Obesity and Diabetes is publishing the articles on type 1 diabetes mellitus, type 2 diabetes mellitus, gestational diabetes, cardio vascular research, osteoporosis, inflammation, cancer, molecular and cellular mechanisms, obesity and diabetes therapy, diabetic drugs, genetics of obesity and diabetes etc. Articles published in this journal are subjected to rigorous peer review and revision process before being accepted for publication, to maintain quality and the standards set for academic journals. This Journal ensures the barrier-free distribution of its content through online free access and thus helps in improving the citations for authors

Copyright: (C2014 Yamaguchi. This is an open-access article distributed under the terms of the Creative Commons Attribution License, which permits unrestricted use, distribution, and reproduction in any medium, provided the original author and source are credited. and attaining a good impact factor. This Journal is one of the best Open Access journals of Scholarly publishing.

Integrative Obesity and Diabetes will accept original research articles, review articles, editorial, case reports and short communication on all the aspects of endocrinology, obesity and diabetes for review and possible publication. The journal is using Editorial Tracking System for quality in review process. Editorial Tracking is an online manuscript submission, review and tracking systems. Review processing will be performed through the editorial board members of the Journal or outside experts; at least two independent reviewers approval followed by editor approval is required for acceptance of any citable manuscript. This is done in accord with the highest standards of professional ethics. Authors may submit manuscripts and track their progress through the system, hopefully to publication.

This Journal covers integrative fields of nutrition, cytokines, hormones, inflammation, classical childhood obesity, obesity causes and disorders, morbid obesity, obesity diabetes, endocrine disorders, obesity and weight loss, obestiy adolescence, metabolic syndrome, types of diabetes, insulin resistant, gestational diabetes, exocrine pancreatic disease, growth hormone excess, diabetic retinopathy, diabetic neuropathy, diabetic nephropathy, gastrointestinal disease, osteoporosis, cancer, genetic defects, cocio-cultural, prevention, epidemiology, pathogenesis, medication and treatment, and formulations.

I hope that this Journal will contribute in the development for biomedical research in the clinical fields of obesity and diabetes with valuable information.
Correspondence to: Dr. Masayoshi Yamaguchi, Department of Hematology and Medical Oncology, Emory University School of Medicine, 1365 C Clifton Road, NE, Atlanta, GA 30322, USA, E-mail: yamamasa1155@yahoo.co.jp

Received: August 24, 2014; Accepted: August 26, 2014; Published: September 01,2014 